\title{
Secondary resistance to Cabergoline-pitfalls and challenges of managing macroprolactinoma with high dose dopamine agonist therapy
}

Dr A Abdalaziz ${ }^{1}$, Dr S Nag ${ }^{1}$, Dr B Dhakshinmoorthy ${ }^{1}$

1. James Cook University Hospital, Middlesbrough

\section{Introduction:}

$\checkmark$ Dopamine agonists (DA) are first line therapy for Prolactinoma which normalises prolactin (PRL) level in $80 \%$ of cases at a median weekly dose of $1 \mathrm{mg}$.

$\checkmark$ An accepted criterion of pharmacological resistance to DA is failure to normalize PRL levels.

$\checkmark$ Failure to normalize PRL levels is seen in $24 \%$ of those treated with bromocriptine, $13 \%$ of those treated with pergolide and $11 \%$ of those treated with cabergoline.

$\checkmark$ Although most patients who respond to dopamine agonists with a normalization of PRL levels have substantial tumour size reduction not all do and viceversa.

$\checkmark$ We report a case of aggressive macroprolactinoma that required $7 \mathrm{mg}$ of Cabergoline to reduce prolactin despite radiological evidence of tumour shrinkage.

\section{Case Summary:}

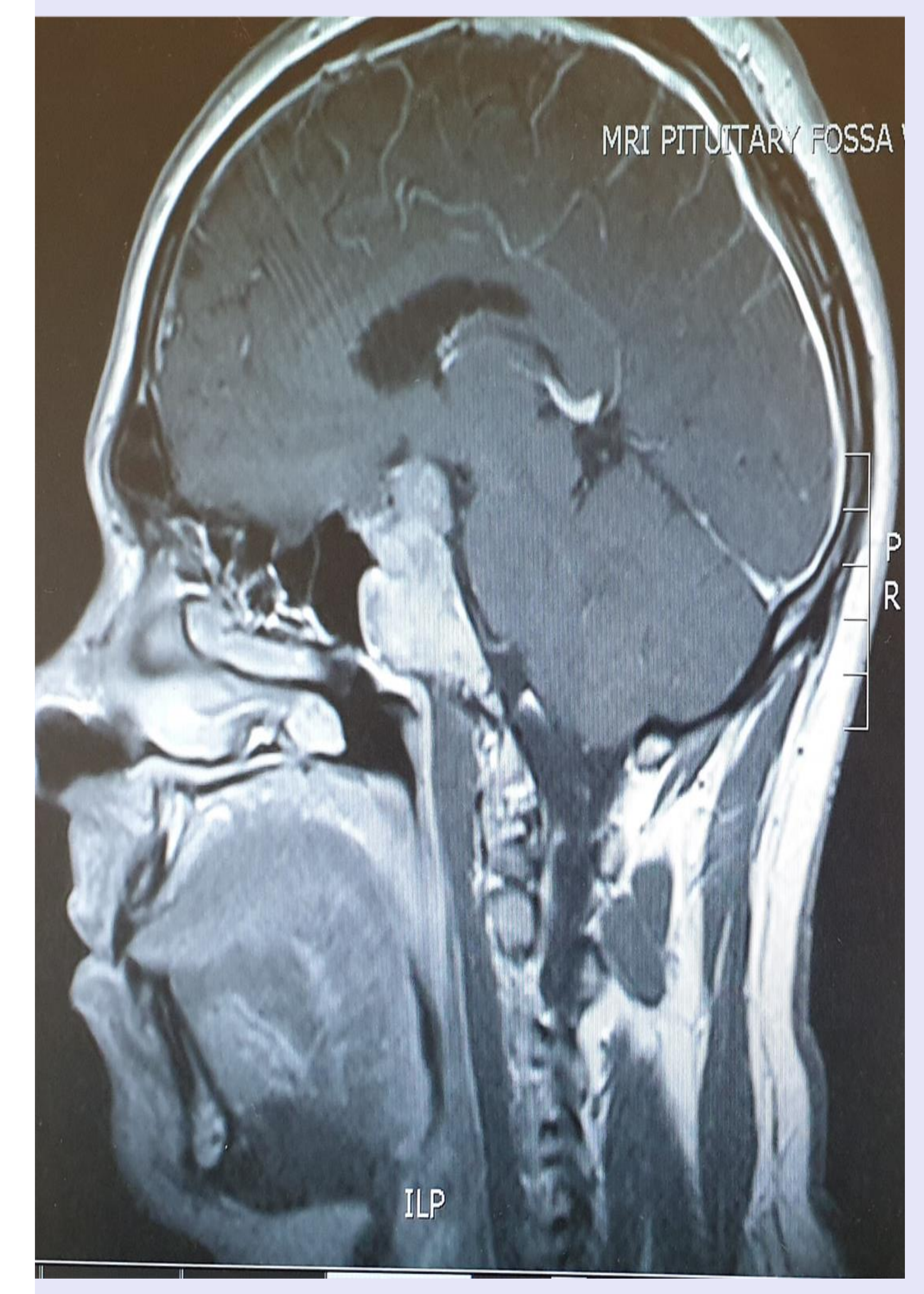

(A) Initial MRI.

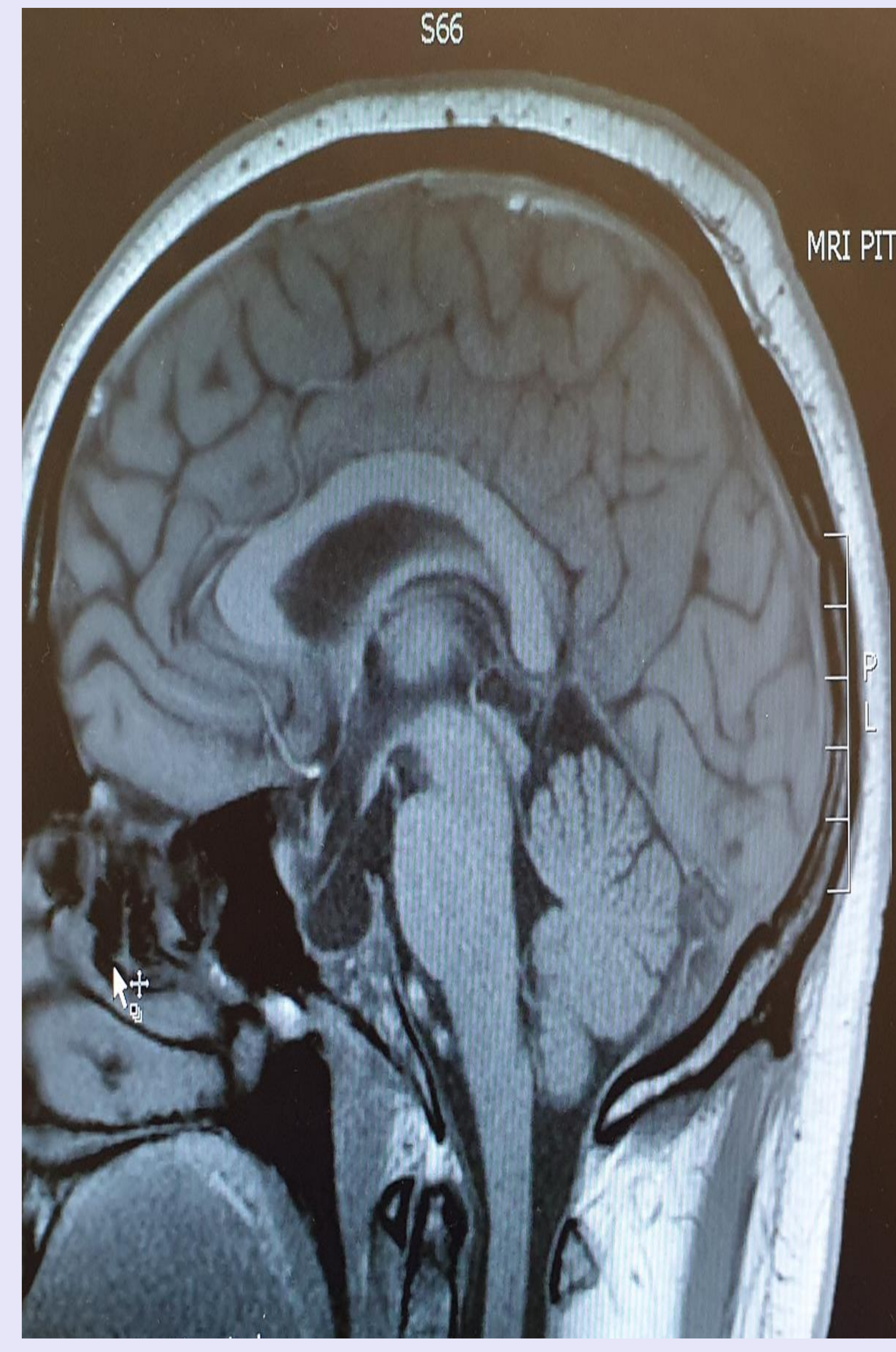

(B) post Cabergoline

\section{Summary:}

- Resistance to dopamine agonists can be defined with respect to failure to normalize PRL levels and failure to decrease tumor size by $\geq 50 \%$

- This case highlights marked secondary resistance to Cabergoline following an initial favourable response.

- Secondary resistance to DA occurs rarely but this case demonstrates that effective tumour shrinkage can be obtained with higher doses of cabergoline with careful monitoring of adverse effects.

- Once tumour shrinkage has been achieved the dose of cabergoline should be reduced to the lowest effective dose that maintains a stable prolactin level.

- Complete normalisation of prolactin may not be feasible or indicated in the majority of cases.

1. Patients and the endocrine team at James Cook University Hospital 